\title{
DÜBLIN
}

Technological University Dublin

ARROW@TU Dublin

Articles

School of Physics \& Clinical \& Optometric

Science

2020

\section{Flash VEP in Clinically Stable Pre-Term and Full-Term Infants}

Anish Kharal

Institute of Medicine, Tribhuvan University, Kathmandu,Nepal

Safal Khanal

University of Alabama atBirmingham, Birmingham, AL, USA

Jyoti Baba Shrestha

Institute of Medicine, Tribhuvan University, Kathmandu,Nepal

See next page for additional authors

Follow this and additional works at: https://arrow.tudublin.ie/scschphyart

Part of the Optometry Commons

\section{Recommended Citation}

Kharal, A., Khanal, S., Shrestha, J.B. et al. Flash VEP in clinically stable pre-term and full-term infants. Doc Ophthalmol (2020). DOI:10.1007/s10633-020-09773-0

This Article is brought to you for free and open access by the School of Physics \& Clinical \& Optometric Science at ARROW@TU Dublin. It has been accepted for inclusion in Articles by an authorized administrator of ARROW@TU Dublin. For more information, please contact arrow.admin@tudublin.ie, aisling.coyne@tudublin.ie, gerard.connolly@tudublin.ie.

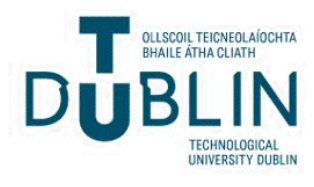




\section{Authors}

Anish Kharal, Safal Khanal, Jyoti Baba Shrestha, Gauri Shankar Shrestha, and Nabin Paudel 


\title{
Flash VEP in clinically stable pre-term and full-term infants
}

\author{
Anish Kharal (D) Safal Khanal (D) Jyoti Baba Shrestha - Gauri Shankar Shrestha • \\ Nabin Paudel $\mathbb{D}$
}

Received: 19 August 2019/Accepted: 20 May 2020

(C) Springer-Verlag GmbH Germany, part of Springer Nature 2020

\begin{abstract}
Purpose Pre-term infants are at risk of abnormal visual development that can range from subtle to severe. The aim of this study was to compare flash VEPs in clinically stable pre-term and full-term infants at 6 months of age.

Methods Twenty-five pre-term and 25 full-term infants underwent flash VEP testing at the age of 6 months. Monocular VEPs were recorded using flash goggles on a RETIscan system under normal sleeping conditions. Amplitude and peak time responses of the P2 component in the two eyes were averaged and compared between the two groups. Multiple regression analyses were performed to assess the relationship of the P2 responses with birth weight (BW) and gestational age (GA).
\end{abstract}

Anish Kharal and Safal Khanal contributed equally to this work and are co-first authors.

A. Kharal · J. B. Shrestha - G. S. Shrestha

B.P. Koirala Lions Centre for Ophthalmic Studies, Institute of Medicine, Tribhuvan University, Kathmandu, Nepal

S. Khanal

School of Optometry, University of Alabama at

Birmingham, Birmingham, AL, USA

N. Paudel $(\square)$

Centre for Eye Research Ireland, Technological

University Dublin, Dublin, Ireland

e-mail: paudel.nabin@gmail.com
Results At 6 months corrected age, pre-term infants had significantly delayed $\mathrm{P} 2$ peak times than full-term infants (mean difference: 10.88 [95\% CI 4.00-17.76] $\mathrm{ms}, p=0.005)$. Pre-term infants also showed significantly reduced P2 amplitudes as compared to fullterm infants (mean difference: 2.36 [0.83-3.89] $\mu \mathrm{V}$, $p=0.003$ ). Although the regression model with GA and BW as fixed factors explained $20 \%$ of the variance in the $\mathrm{P} 2$ peak time $\left(F_{2,47}=5.98, p=.0045\right)$, only GA showed a significant negative relationship $(\beta=-2.66, \quad p=.003)$. Neither GA $(\beta=0.21$, $p=.28)$ nor $\mathrm{BW}(\beta=0.001, p=.32)$ showed any relationship with $\mathrm{P} 2$ amplitude.

Conclusions Our results demonstrate that, compared with full-term infants, clinically stable pre-term infants exhibit abnormal flash VEPs, with a delay in P2 peak time and a reduction in P2 amplitude. These findings support a potential dysfunction of the visual pathway in clinically stable pre-term infants as compared to full-term infants.

Keywords Flash VEP · Full-term · Infants · Preterm $\cdot$ Visual evoked potential

\section{Introduction}

Approximately one in ten babies are born pre-term every year [1], with the rate of pre-term birth ranging 
from 5 to $18 \%$ across different regions of the world [2]. There is an extensive amount of literature on the effect of pre-term birth on visual [3,4] and neurological development [5]. Pre-term children are at a higher risk of abnormal visual and neurological development than their full-term peers. Ocular and visual deficits such as retinopathy of prematurity, refractive errors and cortical visual impairment are common in pre-term infants $[6,7]$. Mild-to-severe structural alterations of several brain areas including the cortical and extracortical visual areas are also observed in pre-term infants [8].

Visual evoked potential (VEP) is a minimally invasive investigative procedure that provides a functional probing of the visual pathway in infants and has long been used to explore functional deficits in the visual pathway of the pre-term brain [9]. VEP is particularly useful to investigate the functional integrity of the visual system of high-risk infants where obvious retinal and neurological abnormalities are not observed [10]. Several flash VEP studies of high-risk infants are available in the literature [11-15]. These studies have demonstrated that immature P2 waveform [11], irreproducible waveform responses, and unrecordable VEP measurements [13] are associated with abnormal neurodevelopment [12] and visual impairment in pre-term infants. VEP has also been investigated for its prognostic value of long-term visual and neurodevelopmental outcomes in infants [16-18] but the results are ambiguous. While most studies have demonstrated that early flash VEP in infants is predictive of long-term neurodevelopmental or visual status, some studies have shown contrasting findings [19].

Clinically or medically stable pre-term infants (infants with no signs of infection, severe desaturation and bradycardia, and not requiring invasive respiratory support) are also considered to be at high risk of abnormal neurodevelopment [5] and can have several neurological insults [20]. However, very little is understood on whether these infants differ from their full-term counterparts with regard to the development and maturation of the visual pathway. In this study, we sought to investigate the effect of pre-term birth on visual cortical pathway as assessed with flash VEP. We also explored any presence of refractive errors in this specific population.

\section{Methods}

Participants

Twenty-five pre-term ( 18 males and 7 females) and 25 full-term infants (13 males and 12 females) participated in this study. Infants were recruited from the Paediatric Department of Tribhuvan University Teaching Hospital and Paediatric Ophthalmology unit of B.P. Koirala Lions Centre for Ophthalmic Studies in Kathmandu, Nepal. All parents provided written informed consent for their children to participate in the study. The institutional ethical review committee of the Institute of Medicine provided ethical approval for the study. Pre-term infants were eligible for participation if they were clinically stable and had (1) Gestational age (GA) of 37 weeks or younger, (2) Birth weight (BW) less than $2500 \mathrm{~g}$, (3) no systemic complications of meningitis, cerebral palsy, brain damage, birth asphyxia, stroke, congenital malformations, genetic or chromosomal abnormalities and (4) absence of congenital cataract, congenital glaucoma, retinopathy of prematurity or other major complications. Out of the 25 pre-term infants, five were very pre-term (GA $<32$ weeks), 20 were moderate to late pre-term (GA between 32 and 37 weeks), four had very low BW $(1000$ to $<1500 \mathrm{~g})$ and 21 had low BW $(1500$ to $<2000 \mathrm{~g})$. Infants were categorised based on the WHO criteria [21]. None of the pre-term infants had extremely low BW $(<1000 \mathrm{~g})$. To facilitate the comparison with a control group, the study included healthy age-matched infants born at term. All of these full-term infants had (1) GA greater than 37 weeks; (2) birth weight appropriate for GA and more than $2500 \mathrm{~g}$; (3) normal infant vital parameters at the time of birth; and (4) APGAR score (Appearance, Pulse, Grimace, Activity, Respiration) greater than 7 at the time of birth.

\section{Study protocol}

Neonatal and antenatal history of the infants, including birth parameters, were obtained from their medical records. All infants were enrolled consecutively for cross-sectional measurements. Pre-term infants were examined at 6 months ( \pm 1 week) of their corrected age, whereas full-term infants were examined at 6 months ( \pm 1 week) of their chronological age. All examinations were conducted in the presence of 
parents or caretakers of infants. For all participating infants, a paediatric ophthalmologist performed detailed ophthalmic examination (anterior and posterior segment) to determine the ocular health, while a qualified optometrist conducted the measurements of refractive error and VEP recordings. Pupils of both eyes were dilated using a cycloplegic (1\% Cyclopentolate Hydrochloride) eye drop instilled 3 times at an interval of $10 \mathrm{~min}$. Refractive error was measured by performing objective retinoscopy at a distance of $50 \mathrm{~cm}$ after $30 \mathrm{~min}$ from the time of the last instillation of cycloplegic drug. Refractive error was classified as myopia when the spherical equivalent refractive error (SER) was $\leq-0.50$ and as hyperopia when SER was $\geq+0.50 \mathrm{D}$. All procedures in the study adhered to the tenets of the Declaration of Helsinki.

\section{Flash VEP recording}

All infants underwent VEP tests on a separate session to that of retinoscopy under normal deep sleep state (i.e. regular breathing, eyes closed with no eye movements, no spontaneous activities except startles or jerky movements) while laying on their parent's lap. None of these infants received any medication (e.g. sedation/analgesia). Responses were recorded using standard gold-plated cup electrodes $(10 \mathrm{~mm}$, with $2 \mathrm{~mm}$ centre hole) on a RETIscan system (Roland Consult, Germany) in accordance with the ISCEV clinical flash VEP standard [22] other than deviations as mentioned below. Active electrode was placed on the occipital scalp, $2 \mathrm{~cm}$ above the inion according to the International 10/20 electrode placement system (10\% of the nasion-to-inion separation). Reference electrode was placed on the frontal scalp, and ground electrode on the parietal scalp. Retinal stimulation was done monocularly over closed eyelids using light emitting diode flash goggles that delivered red flashes of strength 3 photopic $\mathrm{cd} \mathrm{s} / \mathrm{m}^{2}$ every $30 \mathrm{~s}(2 \mathrm{~Hz})$. The shield provided by the goggles ensured no light entered the unstimulated eye, which was occluded throughout the recording period. No topical eye drops were instilled during VEP measurements. The skin was prepared using an abrasive cream (NuPrep, Weaver and Company, USA) to reduce the resistance prior to the application of the electrodes on the scalp with conductive paste (TEN20, Weaver and Company, USA). During the VEP recordings, impedance for each electrode was maintained at less than $5 \mathrm{k} \Omega$ and band pass filters set at -0.5 to $50 \mathrm{~Hz}$. An in-built automatic artefact rejection algorithm was used to reject blink artefacts above a threshold of $90 \%$ of the range. In the event of excessive artefacts, the trial was rejected and the measurement repeated. VEPs were obtained in two trials, each with 100 measures, and averaged. The built-in RETIport software automatically displayed cursors corresponding to the peaks and troughs of the VEP waveforms. In addition, the software allowed manual repositioning of the cursors. One of the investigators, who has several years of experience with VEP examination, reviewed all waveforms retrospectively and manually shifted cursor positions on occasional cases of misalignment. The same investigator assessed the reproducibility of waveforms of the two trials in each infant by visual inspection immediately after completion of the tests. Subjects with variable trails underwent repeat testing on the following day or were excluded. Altogether, VEPs from 5 pre-term and 3 full-term infants were discarded because of instrument-related (excessive artefacts, high impedance) and subject-related factors (lack of co-operation and lost follow-up for repeat testing), leaving data from 25 pre-term and 25 full-term subjects available for analysis. Previous studies have shown P2 component to be the most prominent and robust component of flash VEP [15, 23]. Therefore, following the completion of the tests, $\mathrm{P} 2$ parameters (amplitudes and peak times) were obtained using a built-in RETIport software v4.8.1.12 and compared between the groups. Peak time was calculated as the time from stimulus onset to the peak of the P2 component, whereas P2 amplitude was measured from the preceding negative peak N2 to the positive peak P2 (Fig. 1).

\section{Statistical analysis}

Data were analysed in R [24] and figures were created using the package ggplot2 [25]. Spherical equivalent refractive error (SER) was calculated as sphere + $\left(\frac{c y l i n d e r}{2}\right)$ in dioptres $(D)$. Significant correlations were observed between data in right eye and left eye (ICC, SER: 0.92 [0.87,0.96], $p<0.0001 ; \mathrm{P} 2$ peak time: 0.85 [0.73, 0.91], $p<0.0001, \mathrm{P} 2$ amplitude: 0.71 [0.54, $0.82], p<0.0001)$. Therefore, average data from two eyes were used for analysis [26]. Normality of the data was tested with Shapiro-Wilk test and verified with 

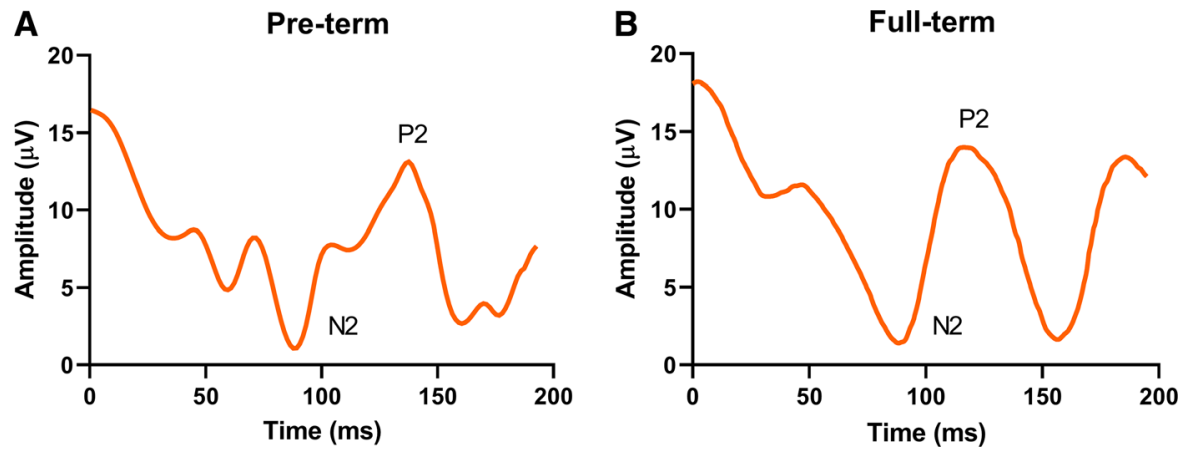

Fig. 1 Flash VEP waveforms showing negative wave N2 and positive wave component P2 from the left eye of a pre-term and a fullterm infant. The waveforms are replotted from software-generated reports

histograms and Q-Q plots. Data on refraction and peak times were normally distributed for both pre-term and full-term cohorts (all $p>0.05$ ), but average amplitude data (mean of two eyes) were not $(p=0.002)$. Therefore, differences between pre-term and full-term infants were tested for significance with WilcoxonMann-Whitney test for independent samples. The two independent variables were only moderately correlated $(r=0.38)$, thus meeting the multicollinearity assumption. General linear models with GA and BW as fixed effects were carried out to investigate whether GA and BW could significantly affect flash VEP outcome variables. Separate models were constructed for P2 peak times and amplitudes. Relationship of residual and predicted values showed that the data were homoscedastic. In addition, distributions of residuals were normal, and there were no influential outliers as assessed with Cook's distance. Data are presented as mean \pm SD and mean difference $[95 \%$ CI], unless otherwise stated. $P$ value of $<0.05$ is considered to be statistically significant. Since P2 amplitudes and peak times were not independent of each other, comparisons of these outcomes between the two groups used Bonferroni-corrected alpha of 0.025 . As limited number of infants were allocated to each cohort based on convenience sampling due to limited resources and short duration of the project, effect sizes and posthoc power calculations for twosided tests at 0.05 significance level were performed.

\section{Results}

Pre-term infants had significantly lower GA (mean difference: 5.51 [4.58-6.45] weeks, $p<0.001$ ) and
BW (mean difference: 808 [627-989] g; $p<0.001$ ) (Table 1). No significant association was observed between gender distribution and infant's birth status (i.e. pre-term or full-term) $\left(\chi^{2}=2.12, p=0.14\right)$.

\section{Refractive Error}

Nine out of 25 (36\%) pre-term infants were myopic, whereas only one out of 25 (4\%) full-term infants were myopic. Average SER was $0.31 \pm 0.99 \mathrm{D}$ in pre-term infants and $0.92 \pm 0.52 \mathrm{D}$ in full-term infants. There was a statistically significant difference in average SER (mean of two eyes) between the pre-term and full-term infants, with the pre-term infants showing a relative bias towards myopia (mean difference $=0.64$ [0.18-1.10], $p=0.03$, effect size $d=0.77$, power $=$ 0.74 , Fig. 2a). Inter-eye difference of mean SER was $0.13 \pm 0.42 \mathrm{D}$ in pre-term infants and $0.05 \pm 0.20 \mathrm{D}$ in full-term infants.

P2 peak times and amplitudes

Pre-term infants showed delayed P2 peak times $(133.3 \pm 12.7 \mathrm{~ms})$ compared to full-term infants $(122.4 \pm 11.4 \mathrm{~ms})$; this difference in peak times was statistically significant (mean difference $=10.88$ [4.00-17.76] $\mathrm{ms}, p=0.005$, effect size $\mathrm{d}=0.90$, power $=0.87$, Fig. 2B). Similarly, pre-term infants demonstrated reduced P2 amplitudes $(6.84 \pm 2.10 \mu \mathrm{V})$ in comparison with the full-term infants $(9.20 \pm 3.18 \mu \mathrm{V})$ with a statistically significant difference in amplitudes (mean difference $=2.36$ [0.83-3.89] $\mu \mathrm{V}, p=0.003$, effect size $\mathrm{d}=0.88$, power $=0.84$, Fig. $2 \mathrm{c}$ ). 
Table 1 Distribution of pre-term and full-term infants in terms of gestational age and birth weight

$N A$ not applicable, $G A$ Gestational age, $B W$ birth weight

*Independent sample $t$ test

\begin{tabular}{|c|c|c|c|c|}
\hline & Pre-term & Full-term & $p$ value & $95 \%$ CI of diff [lower, upper] \\
\hline Frequency $(n)$ & 25 & 25 & & \\
\hline \multicolumn{5}{|l|}{ GA (weeks) } \\
\hline 28 to $<32$ & $5(20 \%)$ & NA & & \\
\hline 32 to $<37$ & $20(80 \%)$ & NA & & \\
\hline$>37$ to 42 & NA & $25(100 \%)$ & & \\
\hline Mean $\pm 95 \%$ CI & $33.83 \pm 0.86$ & $39.34 \pm 0.44$ & $<0.001 *$ & {$[-6.45$ to -4.58$]$} \\
\hline Range & $30.43-37.00$ & $38.00-41.71$ & & \\
\hline \multicolumn{5}{|l|}{ Birth weight (g) } \\
\hline 1000 to $<1500$ & $4(16 \%)$ & & & \\
\hline 1500 to $<2500$ & $21(84 \%)$ & NA & & \\
\hline$\geq 2500$ & NA & $25(100 \%)$ & & \\
\hline Mean $\pm 95 \%$ CI & $1996 \pm 163.27$ & $2804 \pm 97.34$ & $<0.001 *$ & {$[-989$ to -627$]$} \\
\hline Range & $1300-2450$ & $2500-3300$ & & \\
\hline
\end{tabular}

A

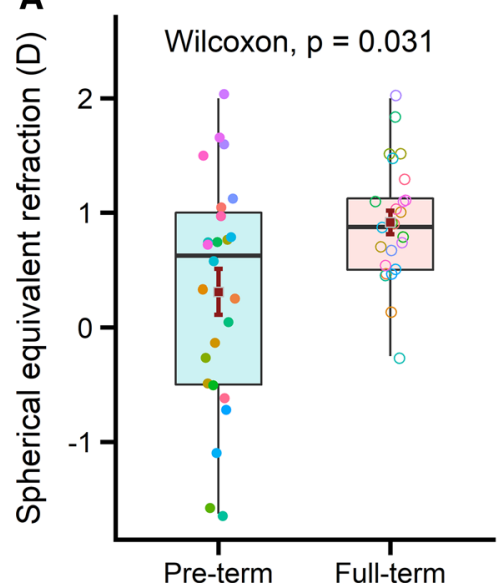

B

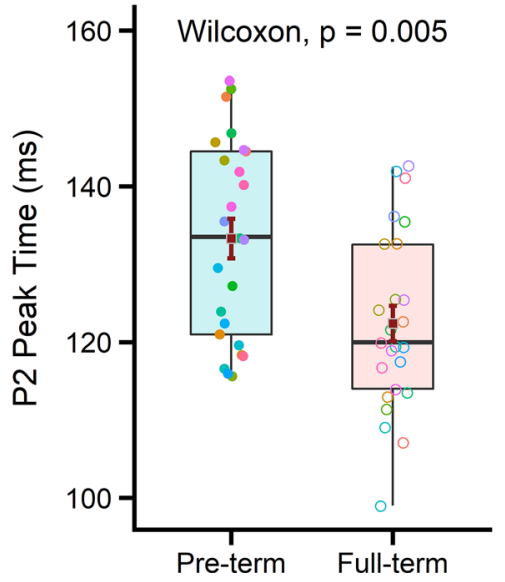

C

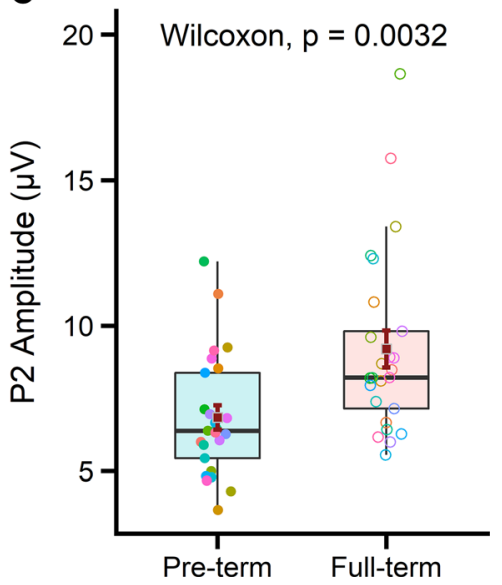

Fig. 2 a Average spherical equivalent refractive error (SER, mean of two eyes), b P2 peak times and c P2 amplitudes of preterm and full-term infants. There were significant differences in SER, P2 peak times and amplitudes between the pre-term and full-term infants. Dark red error bars inside the boxplot represent

\section{Relationship of P2 parameters with GA and BW}

We further explored the relationship of $\mathrm{P} 2$ parameters (peak times and amplitudes) with GA and BW by conducting multiple linear regression analyses, with GA and BW as fixed factors. The results of the regression indicated that the model for the peak time explained $20 \%$ of the variance and that the model was a significant predictor $\left(F_{2,47}=5.98, p=0.0045\right.$, effect size $f^{2}=0.25$, power $=0.88$, Fig. $3 \mathrm{a}$ ). In particular, GA contributed significantly to the model $(\beta=-2.66$, $p=0.003$ ); peak time was delayed by $2.66 \mathrm{~ms}$ for mean \pm SEM. Filled and open-circle symbols represent preterm and full-term infants, respectively. Each colour matches infants in the two cohorts. SER spherical equivalent refraction, $D$ dioptre, $m s$ milliseconds, $\mu V$ microvolts

every one-week reduction in gestational age. However, BW did not have an effect on peak times $(\beta=-0.01$, $p=0.14)$. The final predictive model was: $\mathrm{P} 2$ Peak time $=205.89+(-2.66 * \mathrm{GA})+(0.01 * \mathrm{BW})$.

For the amplitude model, GA and BW only explained $18 \%$ of variance in the P2 amplitudes. Although the multiple regression model was a significant predictor of $\mathrm{P} 2$ amplitudes $\left(F_{2,47}=5.03\right.$, $p=0.01$, effect size $f^{2}=0.22, \quad$ power $=0.83$, Fig. $3 b)$, the fixed effects of both GA $(\beta=0.21$, $p=0.28)$ and $\mathrm{BW}(\beta=0.001, p=0.32)$ were not 


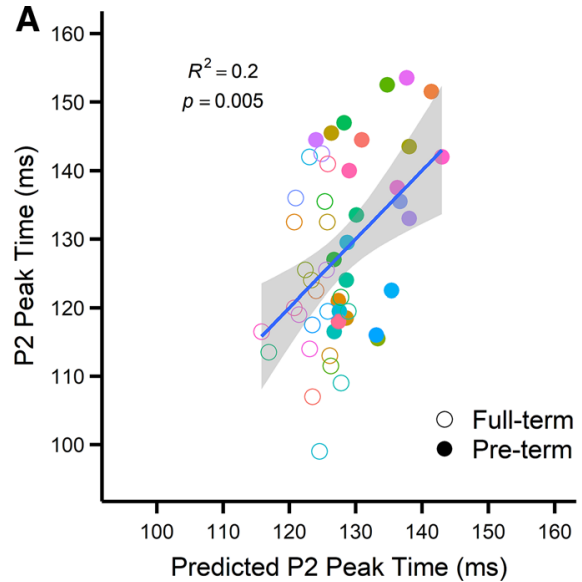

Fig. 3 Average a P2 peak times and b P2 amplitudes (mean of two eyes) as functions of predicted values from fixed-effect models with gestational age (GA) and birth weight (BW) as the fixed factors. The multiple regression model was significant for both the $\mathrm{P} 2$ peak times and amplitudes, but only gestational age

significant. The final predictive model was: P2 Amplitude $=-2.52+0.21 *(\mathrm{GW})+0.001 *(\mathrm{BW})$.

\section{Discussion}

This study evaluated refractive status and flash VEP parameters in a cohort of clinically stable pre-term infants and examined whether these parameters are different when compared with an age-matched cohort of full-term infants. Furthermore, the study explored the relationship between the VEPs with two common birth parameters: GA and BW. Our results indicate that clinically stable pre-term infants may have altered emmetropization [27] since a higher number of preterm infants demonstrated myopic refractive errors than their full-term peers. This finding is consistent with previous studies that suggest children born preterm are at a higher risk of developing myopia [28-30]. With regard to the VEP parameters, pre-term infants had relatively prolonged and diminished VEPs than their full-term counterparts. These findings suggest an abnormal cortical neural activity in preterm infants despite their clinical stability and indicate potential immaturity of the global visual pathway. Our results further show that the birth parameter GA has negative relationship with the VEPs wherein children with shorter gestation period at birth are likely to show altered VEPs with delayed peak times. However, birth

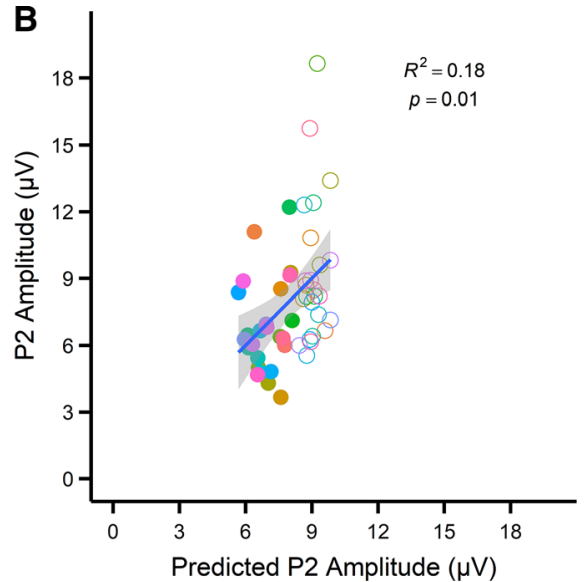

significantly predicted $\mathrm{P} 2$ peak times. The blue lines denote the fit of the multiple regression models. Each coloured dot represents an infant. Filled and open circle symbols represent pre-term and full-term infants, respectively. The grey zones indicate $95 \% \mathrm{CI}$

weight was not significantly related to the VEP parameters (amplitude and peak time) in our cohort. These findings imply that infants with shorter GA, irrespective of their clinical status, may have suboptimal development and compromised integrity of the visual pathway.

Our results of prolonged and reduced VEPs in preterm infants were slightly surprising, given that these infants were clinically stable and had no signs of systemic and ocular morbidities except refractive errors. Previous studies have reported a range of anomalous flash VEPs in infants born pre-term but all of those studies involved children with a range of neurological insults in contrast with our study where none of the children had features of neurological insult. Placzek et al. reported flash VEP findings of 70 preterm infants and demonstrated that $90 \%$ of the neurologically normal infants had distinct P2 waveform found similar to full-term infants from 35 to 36 week's gestation [11]. However, children with neurological impairment and brain insults such as intra-ventricular haemorrhage had immature waveforms. Similar findings were reported by De Vries et al. [12] and Eken et al. [13] who showed pre-term infants with periventricular leukomalacia had abnormal flash VEP waveforms (irreproducible waveforms, unable to record). Nonetheless, a growing body of literature now suggests an increased vulnerability of visual cortical function [10], global motion processing, [31] and visual 
sensory and perceptual functioning [32] in infants born pre-term even in the absence of manifest oculo-visual and systemic abnormalities. In a sweep VEP study, Hou et al. studied visual cortical functions in 52 term infants and 58 very low birth weight and shortgestation infants without significant retinopathy of prematurity or neurologic morbidities [10]. They showed increased threshold and reduction in amplitudes for spatial frequency, contrast, and vernier displacement measures in pre-term infants at 5-7 months of corrected age when compared with full-term infants. Hou et al. concluded that pre-term babies, even in the absence of identifiable retinal or neurologic abnormalities, demonstrate reduced visual cortical sensitivity. More recently, Michalczuk et al. [33] evaluated pattern VEPs in 25 pre-term and 28 term born school age children and demonstrated reduced P100 amplitudes and delayed P100 peak times in preterm born children compared with those born at term. Although different forms of VEPs were used to probe the functionalities of the visual system in the studies by Chou et al. and Michalczuk et al., their findings are in line with our results in demonstrating that high-risk infants even if they do not show any obvious clinical visual or systemic disorders can still have altered visual pathway.

Further, and perhaps stronger, evidence comes from another closely related study in which Feng et al. [14] tracked flash VEPs in pre-term and full-term infants from 1 to 18 months following birth and demonstrated significantly prolonged P2 peak times and reduced P2 amplitudes in pre-term infants compared with full-term controls. At 6-month corrected age, the mean P2 peak times of very low birth weight infants, low birth weight infants, and full-term infants were $141 \pm 19 \mathrm{~ms}, 135 \pm 16 \mathrm{~ms}$ and $130 \pm 7 \mathrm{~ms}$, respectively. These values are slightly higher than those observed in our cohort of pre-term $(133.3 \pm 12.7 \mathrm{~ms})$ and full-term children (122.4 $\pm 11.4 \mathrm{~ms})$. This discrepancy could be due to several reasons. First, there were considerable differences in the stimulation protocol and data extraction process between this study and the study by Feng et al., such as the use of band pass filter $(0.5-50 \mathrm{~Hz}$ vs $1-100 \mathrm{~Hz})$, the averaging of the waveforms (100 vs 50-100) and the stimulus colour and intensity (red, $3 \mathrm{~cd} \mathrm{~s} \mathrm{~m}^{-2}$, vs unspecified). Second, the sample size in Feng et al.'s study was larger than ours with considerably higher number of infants in each of the three groups (20 very low birth weight, 42 low birth weight and 41 full-term). In contrast, we only had two groups: 25 pre-term and 25 full-term infants. As there were only 4 children in the very low birth weight group, we combined these infants into the low birth weight group, which resulted in a preponderance of pre-term infants with low birth weight. Interestingly, the mean P2 peak time of the low birth weight infant group in Feng et al.'s study [14] was comparable to the P2 peak time of pre-term infants in this study. Another study by Feng et al. [15] reported similar findings of delayed $\mathrm{P} 2$ peak time in pre-term infants as compared with term infants at the corrected age of 2 years, but the differences were only significant between the very low birth weight infants and the control or term infants. More longitudinal studies are warranted to unambiguously establish the effect of these subtle deficits on the visual pathway of pre-term infants for long-term clinically significant visual outcomes.

Abnormal flash VEPs (amplitude reduction and peak time delay) in clinically stable pre-term infants as observed in our study could in part be attributed to the speed at which the myelination process occurs. Myelination is a salient feature in neural conduction and cortical development. It commences before birth and progresses rapidly in the first few months of life [34] as evident by the detection of traces of myelin until 1-2 months of age on T1-weighted MRI [35]. The rates of myelination could vary between infants born with complete gestational period and those born pre-term, resulting in lengthening of the peak times and amplitude drop as observed in the pre-term infants relative to the full-term infants.

This study has a few limitations. Although our findings demonstrate subtle differences in VEPs between pre-term and full-term infants at 6 months of age, we are unable to generalise the findings beyond this period due to the cross-sectional nature of the study. Inferences about the cortical responses were obtained by simply analysing the P2 component response of the flash VEP. While the P2 component is the most prominent feature of the flash VEP [15] and better relates to the amount of functional neural fibres and conduction velocity [36], sensory signal integration and developmental changes in the afferent pathways are likely to be a complex process. Finally, our sample size was too small for the stratification of the results according to the distribution of BW and GA. Moreover, the small sample size may also limit 
our findings to be generalised to all clinically stable pre-term infants although power calculation demonstrated that our study had sufficient power.

In conclusion, pre-term infants, despite being clinically stable, show prolonged and reduced VEPs compared with the full-term infants and may be at risk for a slower maturation and a compromised functional integrity of the visual pathway. The implications of these findings for later visual developmental outcomes remain to be investigated. Further longitudinal studies would aid in the understanding of the relationship between these early VEP parameters with the visual developmental outcomes of clinically stable pre-term infants later in life.

Funding No funding was received for this research.

\section{Compliance with ethical standards}

Statement of human rights All procedures in human participants were performed in accordance with the ethical standards of the Institutional Review Board of the Institute of Medicine, Tribhuvan University and with the 1964 Helsinki Declaration and its later amendments or comparable ethical standards.

Statement on the welfare of animals This article does not contain any studies with animals performed by any of the authors.

Informed consent Informed consent was obtained from parents of all individual participants included in the study.

Conflict of interest None of the authors listed in this manuscript have any conflict of interest.

\section{References}

1. Beck S, Wojdyla D, Say L et al (2010) The worldwide incidence of pre-term birth: a systematic review of maternal mortality and morbidity. Bull World Health Organ 88:31-38

2. Vogel JP, Chawanpaiboon S, Moller AB et al (2018) The global epidemiology of pre-term birth. Best Pract Res Clin Obstet Gynaecol 52:3-12

3. Leung MPS, Thompson B, Black J et al (2018) The effects of pre-term birth on visual development. Clin Exp Optom 101:4-12

4. Madan A, Jan JE, Good WV (2005) Visual development in pre-term infants. Dev Med Child Neurol 47:276-280

5. Arpino C, Compagnone E, Montanaro ML et al (2010) Preterm birth and neurodevelopmental outcome: a review. Childs Nerv Syst 26:1139-1149

6. O'Connor AR, Wilson CM, Fielder AR (2007) Ophthalmological problems associated with pre-term birth. Eye 21(10):1254-1260
7. Cooke RWI, Foulder-Hughes L, Newsham D, Clarke D (2004) Ophthalmic impairment at 7 years of age in children born very pre-term. Arch Dis Child Fetal Neonatal Ed 89:F249-F253

8. Leijser LM, de Bruïne FT, Steggerda SJ et al (2009) Brain imaging findings in very pre-term infants throughout the neonatal period: part I. Incidences and evolution of lesions, comparison between ultrasound and MRI. Early Hum Dev 85:101-109

9. Taylor MJ, Saliba E, Laugier J (1996) Use of evoked potentials in pre-term neonates. Arch Dis Child 74:70-76

10. Hou C, Norcia AM, Madan A et al (2011) Visual cortical function in very low birth weight infants without retinal or cerebral pathology. Investig Ophthalmol Vis Sci 52:9091-9098

11. Placzek M, Mushin J, Dubowitz LMS (1985) Maturation of the visual evoked response and its correlation with visual acuity in pre-term infants. Dev Med Child Neurol 27:448-454

12. de Vries L (1996) Neurological assessment of the pre-term infant. Acta Paediatr 85:765-771

13. Eken $P$, van Nieuwenhuizen O, van der Graaf Y et al (1994) Relation between neonatal cranial ultrasound abnormalities and cerebral visual impairment in infancy. Dev Med Child Neurol 36:3-15

14. Feng JJ, Wang WP, Guo SJ et al (2013) Flash visual evoked potentials in pre-term infants. Ophthalmology 120:489-494

15. Feng JJ, Wang TX, Yang CH et al (2010) Flash visual evoked potentials at 2-year-old infants with different birth weights. World J Pediatr 6:163-168

16. Shepherd AJ, Saunders KJ, McCulloch DL, Dutton GN (1999) Prognostic value of flash visual evoked potentials in pre-term infants. Dev Med Child Neurol 41:9-15

17. Clarke MP, Mitchell KW, Gibson M (1997) The prognostic value of flash visual evoked potentials in the assessment of non-ocular visual impairment in infancy. Eye 11:398-402

18. Ekert PG, Keenan NK, Whyte HE et al (1997) Visual evoked potentials for prediction of neurodevelopmental outcome in pre-term infants. Neonatology 71:148-155

19. Kato T, Watanabe K (2006) Visual evoked potential in the newborn: does it have predictive value? Semin Fetal Neonatal Med 11:459-463

20. Sorensen LC, Greisen G (2009) The brains of very pre-term newborns in clinically stable condition may be hyperoxygenated. Pediatrics 124:e958-e963

21. World Health Organization (2018) Pre-term birth. Retrieved from https://www.who.int/news-room/fact-sheets/detail/ pre-term-birth

22. Odom JV, Bach M, Brigell M, ISCEV standard for clinical visual evoked potentials: (2016 update) (2016) ISCEV standard for clinical visual evoked potentials:(2016 update). Documenta Ophthalmologica 133(1):1-9

23. Benavente I, Tamargo P, Tajada N et al (2005) Flash visually evoked potentials in the newborn and their maturation during the first six months of life. Doc Ophthalmol 110:255-263

24. R Core Team (2013). R: a language and environment for statistical computing. R Foundation for Statistical Computing, Vienna, Austria. http://www.R-project.org/

25. Wickham H (2016) ggplot2: elegant graphics for data analysis. Springer, New York 
26. Armstrong RA (2013) Statistical guidelines for the analysis of data obtained from one or both eyes. Ophthalmic Physiol Opt 33:7-14

27. Saunders KJ, McCulloch DL, Shepherd AJ, Wilkinson AG (2002) Emmetropisation following pre-term birth. Br J Ophthalmol 86:1035-1040

28. Quinn GE, Dobson V, Kivlin J et al (1998) Prevalence of myopia between 3 months and $51 / 4$ years in pre-term infants with and without retinopathy of prematurity. Ophthalmology 105:1292-1300

29. Choi MY, Park IK, Yu YS (2000) Long term refractive outcome in eyes of pre-term infants with and without retinopathy of prematurity: comparison of keratometric value, axial length, anterior chamber depth, and lens thickness. Br J Ophthalmol 84:138-143

30. Uprety S, Morjaria P, Shrestha JB, Shrestha GS, Khanal S (2017) Refractive status in nepalese pre-term and full-term infants early in life. Optom Vis Sci 94(10):957-964

31. Taylor NM, Jakobson LS, Maurer D, Lewis TL (2009) Differential vulnerability of global motion, global form, and biological motion processing in full-term and pre-term children. Neuropsychologia 47:2766-2778
32. Geldof CJA, Oosterlaan J, Vuijk PJ et al (2014) Visual sensory and perceptive functioning in 5-year-old very preterm/very-low-birthweight children. Dev Med Child Neurol 56:862-868

33. Michalczuk M, Urban B, Chrzanowska-Grenda B, et al (2015) An influence of birth weight, gestational age, and apgar score on pattern visual evoked potentials in children with history of prematurity. Neural Plast 2015

34. Tsuneishi S, Casaer P (1997) Stepwise decrease in VEP latencies and the process of myelination in the human visual pathway. Brain Dev 19:547-551

35. Dietrich RB, Bradley WG, Zaragoza EJ IV et al (1988) MR evaluation of early myelination patterns in normal and developmentally delayed infants. Am J Roentgenol 150:889-896

36. Tobimatsu S, Celesia GG (2006) Studies of human visual pathophysiology with visual evoked potentials. Clin Neurophysiol 117:1414-1433

Publisher's Note Springer Nature remains neutral with regard to jurisdictional claims in published maps and institutional affiliations. 